\title{
Induction of apoptosis by FFJ-5, a novel naphthoquinone compound, occurs via downregulation of PKM2 in A549 and HepG2 cells
}

\author{
XIAOLI WEI, MING LI, MINGMING MA, HUINA JIA, YU ZHANG, \\ WENYI KANG, TIANXIAO WANG and XIAOYAN SHI
}

Institute of Traditional Chinese Medicine, College of Pharmacy, Henan University, Kaifeng, Henan 475004, P.R. China

Received August 10, 2015; Accepted November 10, 2016

DOI: $10.3892 / \mathrm{ol} .2016 .5522$

\begin{abstract}
Pyruvate kinase isoenzyme M2 (PKM2) has previously been identified as a tumor biomarker and as a potential target for cancer therapy. In this study, F§FJ-5, a characterized naphthoquinone modifier of mollugin, was synthesized in order to investigate its anticancer activity and the potential mechanisms. It was observed that FFJ-5 inhibited the cell growth of human lung adenocarcinoma cells A549 and human hepatoma cells HepG2 by MTT assays. FFJ-5 arrested cell cycle at the G2/M phase. Further analyses demonstrated that FFJ-5 attenuated the expression of PKM2 and reduced the production of adenosine triphosphate (ATP). Reduced expression and activity of epidermal growth factor receptor (EGFR) and Akt were observed in A549 and HepG2 cells exposed to FFJ-5. FFJ-5 exposure also resulted in cell apoptosis, in association with decreased intracellular $\mathrm{pH}$ level and mitochondrial membrane potential. In addition, FFJ-5 activated the caspase- 3 cascade. In conclusion, FFJ-5 inhibited cancer cell growth via the blocking the EGFR-Akt-PKM2 pathway or through the synergistic action of EGFR, Akt and PKM2 proteins, alongside a decrease in ATP production. In addition, FFJ-5 induced cancer cell apoptosis by decreasing the intracellular $\mathrm{pH}$ level and the mitochondrial apoptosis pathway. The present results suggest a potential role of FFJ-5 on the therapy of human cancer.
\end{abstract}

Correspondence to: Professor Tianxiao Wang or Dr Xiaoyan Shi, Institute of Traditional Chinese Medicine, College of Pharmacy, Henan University, Jinming Street, Kaifeng, Henan 475004, P.R. China

E-mail:wtx1975@126.com

E-mail: shisheep@126.com

Key words: naphthoquinone modifier, cancer, pyruvate kinase isoenzyme M2, apoptosis

\section{Introduction}

Cancer has long been threatening human health; thus, it is urgent to identify novel effective therapeutic agents and methods for the treatment of cancer. Previous studies have demonstrated that the extracts of madder, a type of traditional Chinese medicine, may be associated with a reduced risk of certain cancers (1). Among these extracts, the anticancer activity of mollugin has been shown to be strong in various types of human cancer cells (2).

Cancer cells produce the majority of their energy via the glycolytic pathway under aerobic conditions (known as the Warburg effect), which is a feature of the majority of cancer cells $(3,4)$. The Warburg effect in cancer cells, caused by dysfunctional oxidative phosphorylation, sustains glycolysis, which is responsible for tumor initiation, progression and metastasis $(5,6)$. Consequently, the molecular basis of aerobic glycolysis in cancer has been biochemically investigated, and deregulated metabolism is gaining recognition as a hallmark of cancer cells, and is being explored for therapeutic potential.

It is widely accepted that specific expression of pyruvate kinase isoenzyme M2 (PKM2) in cancer cells contributes to this aerobic glycolysis phenotype (7). Pyruvate kinase converts phosphoenolpyruvate into pyruvate, catalyzing the rate-limiting step of glycolysis (8). The M1 isoenzyme of pyruvate kinase is present in adult tissues, whereas PKM2 is a spliceosome variant detected in embryonic and cancer cells. PKM2 expression in malignant cells is the result of the tumor microenvironment, and is responsible for maintaining a glycolytic phenotype (9). PKM2, which has been identified as the predominant cause of the Warburg effect in cancer cells, is essential in cancer metabolism and growth $(8,10)$.

The present study revealed that FFJ-5, a characterized naphthoquinone modifier of mollugin, possessed anticancer activities and induced cell apoptosis (2). It was observed that FFJ-5 inhibited cancer cell proliferation, exerting its anticancer effects via inhibiting the expression of PKM2.

\section{Materials and methods}

Antibodies, reagents and test compounds. Rabbit monoclonal anti-PKM2 (1:1,000; catalog no. 4053), rabbit monoclonal 
anti-caspase-3 (1:1,000; catalog no. 9665), rabbit monoclonal anti-cleaved caspase-3 (Asp175; 1:1,000; catalog no. 9664), rabbit monoclonal anti-poly (ADP-ribose) polymerase (PARP) (1:1,000; catalog no. 9532), rabbit anti-human cleaved PARP (Asp214; 1:1,000; catalog no. 5625), mouse monoclonal anti-phosphorylated (p)-epidermal growth factor receptor (EGFR) (p-Tyr1068-EGFR) (1:1,000; catalog no. 2236), rabbit monoclonal anti-Akt (1:1,000; catalog no. 4685) and rabbit monoclonal anti-p-Akt (p-Ser473-Akt) (1:1,000; catalog no. 4060) primary antibodies were obtained from Cell Signaling Technology, Inc. (Danvers, MA, USA). Rabbit polyclonal anti-EGFR (1:1,000; catalog no. 18986-1-AP) and rabbit polyclonal anti- $\beta$-actin $(1: 2,000$; catalog no. 20536-1-AP) primary antibodies, and peroxidase-conjugated AffiniPure goat anti-rabbit/mouse immunoglobulin $\mathrm{G}$ (IgG) secondary antibodies (1:10,000; catalog nos. SA00001-2 and SA00001-1, respectively) were obtained from ProteinTech Group, Inc. (Chicago, IL, USA). EasyScript Two-Step RT-PCR SuperMix kit was obtained from Beijing TransGen Biotech Co., Ltd. (Beijing, China). PrimeScript ${ }^{\mathrm{TM}}$ RT Reagent kit (catalog no. RR047A) and $\mathrm{SYBR}^{\circledR}$ Premix Ex Taq ${ }^{\mathrm{TM}}$ II (catalog no. RR820A) were obtained from Takara Biotechnology Co., Ltd. (Dalian, China). Propidium iodide (PI), RNase A, rhodamine 123 (Rh123) and Hoechst 33342 were obtained from Sigma-Aldrich (Merck Millipore, Darmstadt, Germany). Triton X-100 was obtained from Amresco, LLC (Solon, OH, USA). TRIzol, adenosine triphosphate (ATP) detection kit (catalog no. S0026B) and the pH fluorescent probe 2',7'-bis(2-carboxyethyl)-5-(and-6)-carboxyfluorescein acetoxymethyl ester (BCECF-AM; catalog no. S1006) were obtained from Biyuntian Biotech Co., Ltd. (Shanghai, China). Mollugin (positive control) was purchased from National Institutes for Food and Drug Control (Beijing, China). FFJ-5 (the modified compound of mollugin) was synthesized by Professor Wenyi Kang (College of Pharmacy, Henan University, Henan, China).

Cell culture and cell viability assay. HepG2 (human hepatocellular carcinoma) and A549 (human lung cancer) cells were obtained from the American Type Culture Collection (Manassas, VA, USA). The cells were maintained in Dulbecco's modified Eagle medium (Invitrogen; Thermo Fisher Scientific, Inc., Waltham, MA, USA) supplemented with $10 \%$ fetal bovine serum (Gibco; Thermo Fisher Scientific, Inc.) and $1 \%$ penicillin/streptomycin in a $37^{\circ} \mathrm{C}$ incubator with $5 \%$ $\mathrm{CO}_{2}$. Cell viability was detected by MTT assay. HepG2 and A549 cells were seeded in 96-well plates. After $24 \mathrm{~h}$, the cells were exposed to FFJ-5 for $48 \mathrm{~h}$, using mollugin as a control. Then, the cells were incubated in fresh medium containing MTT (0.5 mg/ml; Sigma-Aldrich; Merck Millipore) at $37^{\circ} \mathrm{C}$ for $4 \mathrm{~h}$, followed by the addition of $150 \mu \mathrm{l}$ dimethyl sulfoxide to replace the medium. The absorbance values at a wavelength of $490 \mathrm{~nm}$ were read with a microplate reader (Tecan Group Ltd., Männedorf, Switzerland).

Western blotting. The cells were harvested in radioimmunoprecipitation assay buffer [50 mM Tris- $\mathrm{HCl}(\mathrm{pH} 8.0), 150 \mathrm{mM}$ sodium chloride, $1.0 \% \mathrm{NP}-40,0.5 \%$ sodium deoxycholate and $0.1 \%$ SDS] with $10 \mu \mathrm{g} / \mathrm{ml}$ of the protease inhibitor phenylmethane sulfonyl fluoride, and the cell lysates were obtained by centrifugation at $12,000 \times \mathrm{g}$ for $10 \mathrm{~min}$. The protein concentration in the cells lysates was determined using a bicinchoninic acid protein assay kit (Beyotime Institute of Biotechnology, Haimen, China), and equal amounts of protein $(40 \mu \mathrm{g})$ were separated by SDS-PAGE. PKM2, EGFR, pEGFR, Akt, pAkt, PARP and cleaved PARP were separated using an $8 \%$ gel. Caspase- 3 and cleaved caspase- 3 were separated using a $12 \%$ gel. Gels were subsequently transferred electrophoretically to a polyvinylidene difluoride membrane (Merck Millipore) at $70 \mathrm{~mA}$ for $2 \mathrm{~h}$. Then, the membrane was blocked in $5 \%$ fat-free milk, and probed with specific primary antibodies against PKM2, EGFR, pEGFR, Akt, pAkt, caspase-3, cleaved caspase-3, PARP and cleaved PARP at $4^{\circ} \mathrm{C}$ overnight. Upon washing in PBS-Tween 20, the membranes were incubated with anti-mouse $\operatorname{IgG}$ or anti-rabbit $\operatorname{IgG}$ secondary antibodies for $2 \mathrm{~h}$ at room temperature. Bands were visualized using an EasyBlot Enhanced Chemiluminescence kit (Sangon Biotech Co., Ltd., shanghai, China) and detected using a FluorChem Q Multifluor System (ProteinSimple, San Jose, CA, USA). $\beta$-actin was used as a loading control.

Reverse transcription-quantitative polymerase chain reaction $(R T-q P C R)$ analysis. Total RNA was isolated using TRIzol. Semi-quantitative PCR was performed using an EasyScript Two-Step RT-PCR SuperMix kit, according to the manufacturer's protocol. A total of $2 \mu \mathrm{g}$ RNA was transcribed into complementary DNA with PrimeScript RT Reagent kit. RT-qPCR was performed with SYBR Premix Ex TaqII, and the PikoReal ${ }^{\mathrm{TM}}$ Real-Time PCR System (Thermo Fisher Scientific, Inc.) was used to measure messenger RNA (mRNA) expression. The following thermocycling conditions were used: $95^{\circ} \mathrm{C}$ for $5 \mathrm{~min} ; 40$ cycles of $95^{\circ} \mathrm{C}$ for $10 \mathrm{sec}, 59^{\circ} \mathrm{C}$ for $30 \mathrm{sec}$ and $72^{\circ} \mathrm{C}$ for $30 \mathrm{sec} ; 60^{\circ} \mathrm{C}$ for $30 \mathrm{sec}$. The reactions for each sample-primer set were performed in triplicate. Relative quantification analysis was performed using the comparative $\mathrm{Cq}\left(2^{-\Delta \Delta \mathrm{Cq}}\right)$ method (11). All data were normalized to the internal control GAPDH. The sequences of the qPCR primers were as follows: PKM2, forward 5'-AGAACTTGTGCGAGC CTCAA-3' and reverse 5'-GAGCAGACCTGCCAGACTC-3' (product length, $128 \mathrm{bp}$ ); and GAPDH, forward 5'-CTCTGC TCCTCCTGTTCGAC-3' and reverse 5'-ACCAAATCCGTT GACTCCGA-3' (product length, 109 bp).

ATP production detection. ATP production was detected using the ATP detection kit, according to the manufacturer's protocol. HepG2 and A549 cells were seeded in 6-well plates. After $12 \mathrm{~h}$, the cells were treated with FFJ-5 $(0,10,20$ or $40 \mu \mathrm{M})$ for $48 \mathrm{~h}$. Then, the cells were washed twice with ice-cold PBS and lysed with ATP lysis buffer. Upon centrifugation at 12,000 x $g$ for $5 \mathrm{~min}$ at $4^{\circ} \mathrm{C}$ to remove cell debris, the supernatant was added to the substrate solution, and the product of the reaction (ATP) was measured using a luminometer (EnSpire ${ }^{\circledR}$; PerkinElmer, Inc., Waltham, MA, USA) by calibration with ATP standards and total protein samples. The ATP production was represented as $\mathrm{nm} / \mathrm{mg}$ protein.

Intracellular $\mathrm{pH}$ detection. Intracellular $\mathrm{pH}$ was analyzed using a confocal scanning laser microscope (FV100-IX81 inverted microscope; Olympus Corporation, Tokyo, Japan). HepG2 and A549 cells were seeded in cell culture dishes for confocal microscopy (glass bottom, 20-mm diameter). After 
$12 \mathrm{~h}$, the cells were treated with FFJ-5 $(0,10,20$ and $40 \mu \mathrm{M})$ for $48 \mathrm{~h}$, and then co-cultured with BCECF-AM $(10 \mu \mathrm{M}$ diluted in culture medium) for $1 \mathrm{~h}$ at $37^{\circ} \mathrm{C}$ in an incubator with $5 \% \mathrm{CO}_{2}$. The cells were subsequently washed with PBS, and the PBS was discarded prior to fluorescence determination. Fluorescence intensity was measured by confocal scanning laser microscopy at $488 \mathrm{~nm}$ excitation and $535 \mathrm{~nm}$ emission. The FV10-ASW software (version 4.2; Olympus Corporation) incorporated in the confocal microscope was used to calculate the fluorescence intensity per $\mu \mathrm{m}^{2}$ automatically, and finally calculated the relative fluorescence intensity of the samples compared with that of the control. Changes in relative fluorescence intensity represent changes in intracellular $\mathrm{pH}$ (12).

Cell cycle analysis. Cell cycle analysis was performed using flow cytometry. HepG2 and A549 cells treated with FFJ-5 $(0,10,20$ or $40 \mu \mathrm{M})$ for $24 \mathrm{~h}$ were collected and washed twice with ice-cold PBS. Cells $\left(1 \times 10^{6}\right)$ were fixed in $75 \%$ ethanol at $4^{\circ} \mathrm{C}$ for $\geq 4 \mathrm{~h}$. Cells were then washed twice with ice-cold PBS, followed by incubation with DNA staining solution [PI $(50 \mu \mathrm{g} / \mathrm{ml})$, RNase A $(50 \mu \mathrm{g} / \mathrm{ml})$ and Triton X-100 (0.5\%)] for $20 \mathrm{~min}$. Cell cycle analysis was immediately performed using flow cytometry (FACSCalibur; BD Biosciences, Franklin Lakes, NJ, USA).

Cell apoptosis analysis. Cell apoptosis was analyzed using an ArrayScan VTI HCS 600-type high-content live cell imaging system (Thermo Fisher Scientific, Inc.). HepG2 and A549 cells were seeded in 96-well plates. After $12 \mathrm{~h}$, the cells were treated with FFJ-5 $(0,10,20$ and $40 \mu \mathrm{M})$ for $48 \mathrm{~h}$, and then washed with ice-cold PBS. Cells were next incubated with $5 \mathrm{mg} / \mathrm{ml}$ Hoechst 33342 for $30 \mathrm{~min}$, and then incubated with $5 \mathrm{mg} / \mathrm{ml} \mathrm{PI}$ for a further $1 \mathrm{~h}$ at $37^{\circ} \mathrm{C}$. The cells were subsequently washed with ice-cold PBS, and cell apoptosis analysis was immediately performed using the aforementioned Array Scan VTI HCS 600-type high-content live cell imaging system. The second channel, which recorded the overall average fluorescence intensity (mean fluorescence intensity), represented the number of cells undergoing late apoptosis.

Mitochondrial membrane potential (MMP) detection. The change in MMP was analyzed using Rh123 staining, and the Rh123 content was measured by confocal scanning laser microscopy (FV100-IX81 inverted microscope; Olympus Corporation). HepG2 and A549 cells were seeded in cell culture dishes for confocal microscopy (glass-bottom, 20-mm diameter). After $12 \mathrm{~h}$, the cells were treated with FFJ-5 $(0,10,20$ and $40 \mu \mathrm{M})$ for $48 \mathrm{~h}$, and then incubated with Rh123 $(5 \mu \mathrm{g} / \mathrm{ml})$ for $30 \mathrm{~min}$ at $37^{\circ} \mathrm{C}$ in and incubator with $5 \% \mathrm{CO}_{2}$. The cells were subsequently washed with PBS, and the PBS was discarded prior to measurement of the fluorescence intensity at $488 \mathrm{~nm}$ excitation and $535 \mathrm{~nm}$ emission. The aforementioned FV10-ASW software was used to calculate the fluorescence intensity per $\mu \mathrm{m}^{2}$ automatically, and finally calculated the relative fluorescence intensity of the samples compared with that of the control. Changes in relative fluorescence intensity represent changes in MMP (13).

Statistical analysis. Each experiment was repeated three times. Relative cell viability is expressed as a percentage relative to that of the untreated control cells. Error bars represent standard deviation. Data were analyzed using analysis of variance for each two-group comparison test. Statistical analyses were conducted using SPSS 17.0 (SPSS, Inc., Chicago, IL, USA). $\mathrm{P}<0.05$ was considered to indicate a statistically significant difference.

\section{Results}

FFJ-5 inhibits cancer cells growth. FFJ-5, a novel naphthoquinone derivative, is a chemically modified compound based on the structure of mollugin (2) (Fig. 1A and B). It was hypothesized that FFJ-5 possessed stronger anticancer activity compared with that of mollugin in human cancer cells. The antiproliferative/survival activity of FFJ-5 in human liver cancer HepG2 cells and human lung cancer A549 cells was investigated and compared with that of mollugin. FFJ-5 induced a significant dose-dependent decrease in cancer cell survival and exhibited a stronger anticancer activity than that of mollugin in HepG2 and A549 cells (Fig. 1C and D). The dose of FFJ-5 required to achieve $50 \%$ cell viability was 25.67 and $20.18 \mu \mathrm{M}$ in HepG2 and A549 cells, respectively.

FFJ-5 downregulates the expression of PKM2. Previous studies had indicated that naphthoquinone derivatives served as specific inhibitors of PKM2 (14). Therefore, in the present study, the effect of FFJ-5 on the mRNA and protein levels of PKM2 was measured in HepG2 and A549 cells. Notably, decreased PKM2 mRNA and protein levels were observed in HepG2 and A549 cells exposed to FFJ-5, in a dose-dependent manner (Fig. 2A and B). In addition, decreased ATP production was observed in HepG2 and A549 cells treated with FFJ-5 (Fig. 2C). These results suggested that FFJ-5 was a valid inhibitor of PKM2, and consequently inhibited cancer cell growth via controlling glycolysis and ATP production in cancer cells.

FFJ-5 inhibits the expression and phosphorylation of EGFR and Akt. Previous studies indicated that the overexpression of PKM2 in solid tumors was due to the activation of EGFR (15). The authors also demonstrated that EGFR induced the nuclear translocation of PKM2 and promoted cell proliferation in liver cancer cells (16). Previous research revealed that reduced or enhanced Akt phosphorylation was accompanied by the downregulation or upregulation of PKM2 protein, respectively (unpublished data). Therefore, in the present study, the effects of FFJ-5 on the expression and phosphorylation of EGFR and Akt were evaluated. A decrease in the total EGFR and Akt protein levels, and a decrease in the phosphorylation of EGFR and Akt, was observed in HepG2 and A549 cells exposed to FFJ-5 (Fig. 3). The results indicated that FFJ-5 may inhibit cancer cell growth via the blocking of the EGFR-Akt-PKM2 signaling pathway or via the synergistic action of EGFR, Akt and PKM2 proteins.

FFJ-5 arrests the cell cycle in the G2/M phase. Several studies reported that PKM2 in cancer cells ensured a correct cell division, so that the depletion of PKM2 would cause uneven distribution of the DNA in the two daughter cells, and consequently would trigger apoptosis in cancer cells (17). The present study revealed that FFJ-5 arrested the cell cycle in 

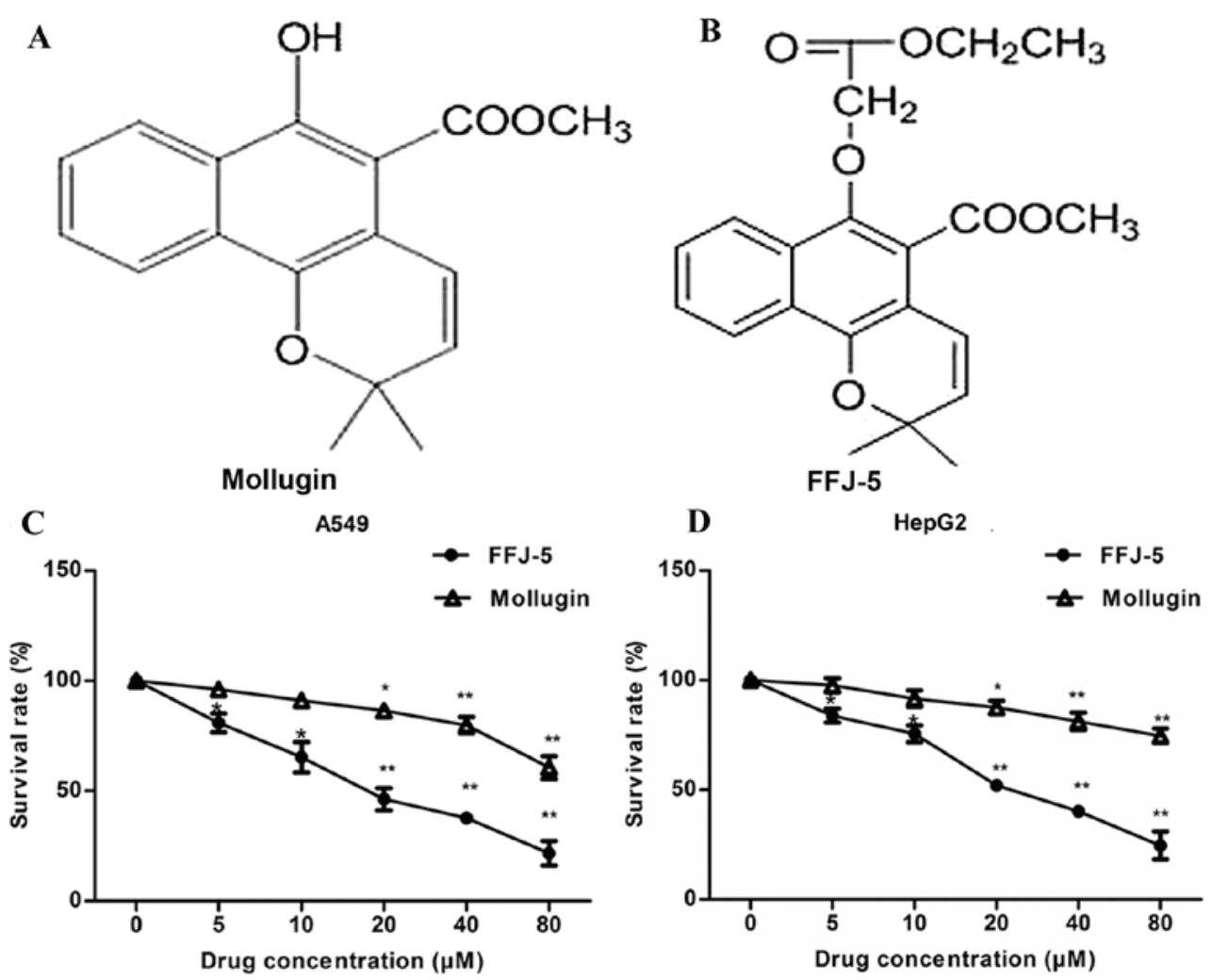

Figure 1. Antiproliferative effects of FFJ-5 and mollugin. Schematic diagram of (A) mollugin and (B) FFJ-5. The antiproliferative effects of FFJ-5 and the positive control mollugin were assessed in (C) A549 and (D) HepG2 cells. Cells were exposed to the compound FFJ-5 and mollugin for $48 \mathrm{~h}$ before being subjected to MTT assay. The data represent the mean and standard deviation obtained from three independent experiments. ${ }^{*} \mathrm{P}<0.05,{ }^{* * *} \mathrm{P}<0.01$.

the G2/M phase in HepG2 and A549 cells, according to flow cytometry analysis (Fig. 4). These results suggested that FFJ-5 may inhibit cancer cell division due to the inhibition of PKM2 and consequently cause programmed cell death or apoptosis.

FFJ-5 induces cancer cells apoptosis. Based on the above results, the possibility that FFJ-5 caused cancer cell apoptosis was evaluated. Cell apoptosis was detected with an Array Scan VTI HCS 600-type high-content live cell imaging system. It was observed that FFJ-5 led to apoptotic cell death in a dose-dependent manner in HepG2 and A549 cells (Fig. 5A). In addition, the change in intracellular $\mathrm{pH}$ in cancer cells exposed to FFJ-5 was also studied. The intracellular $\mathrm{pH}$ was verified by confocal scanning laser microscopy. Decreased intracellular pH level was observed in HepG2 and A549 cells treated with FFJ-5 (Fig. 5B). Additionally, the change in MMP was investigated with Rh123 staining. Increased Rh123 fluorescence intensity was observed in HepG2 and A549 cells following FFJ-5 incubation (Fig. 5C). Proteins associated with the mitochondrial apoptotic pathway were also detected. The western blot results demonstrated that FFJ-5 activated caspase- 3 and consequently induced the cleavage of PARP (Fig. 5D). Taken together, these results supported the induction by FFJ-5 of the apoptotic response in HepG2 and A549 cancer cells.

\section{Discussion}

Tumor patients mostly present with metabolic disorders such as high energy consumption and weight loss (18). In the 1920s, Warburg et al demonstrated that the metabolism of tumor tissues was obviously enhanced and mainly dependent on glycolytic metabolism (19). Sugar consumption speed in tumor cells is greater than that in normal cells. Cancer metabolism characterizes the malignant behavior of tumors. In recent years, the phenomenon of tumor metabolic abnormalities has gained attention, and the Warburg effect became the focus of cancer research (20).

The Warburg effect of cancer cells can promote cancer cell reproduction and invasion (21). The increase in glycolysis results in the acidic microenvironment of cancer (22) that is, the extracellular $\mathrm{pH}$ of cancer cells is lower than their intracellular $\mathrm{pH}$, which can enhance the invasion and proliferation abilities of cancer cells (23). In addition, the acidic microenvironment of cancer cell metabolism can also sustain cell proliferation even under conditions of inadequate nutrition (24). High expression of glycolytic enzymes can aggravate the acidic microenvironment of cancer cells (24).

Sugar metabolic enzymes are closely associated with the cell cycle, and disorders of the cancer cell cycle is one of the mechanisms of carcinogenesis (25). In 2003, a study indicated that the metabolic disorder of cancer cells was associated with the cell cycle regulation of cancer, and that multiple metabolic enzymes were involved in the regulation of histone H2B genes transcription during the cell cycle (26). Therefore, changes in cell metabolism can directly affect the regulation of the cell cycle.

PKM2, a key metabolic enzyme in cancer cells, can promote cancer cell's Warburg effect (27). Numerous studies have demonstrated that PKM2 has a crucial role during the formation and growth of cancer by increasing the acidic 
A
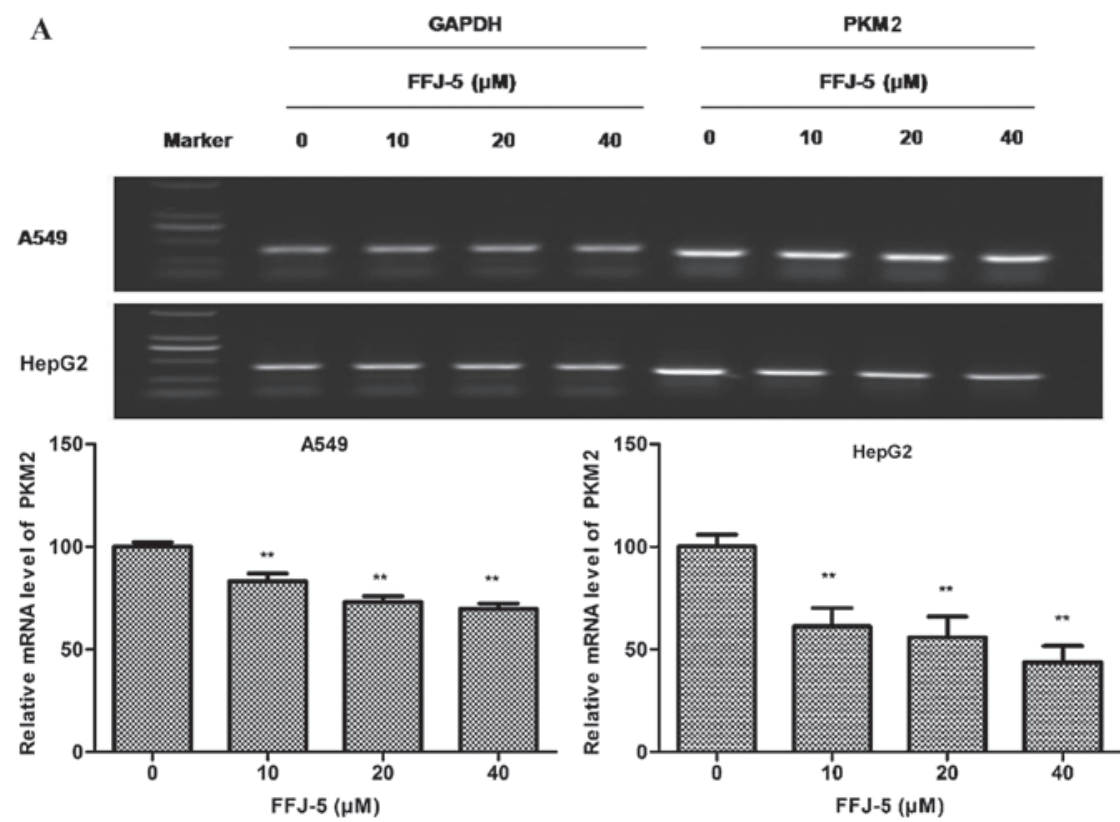

B

A549
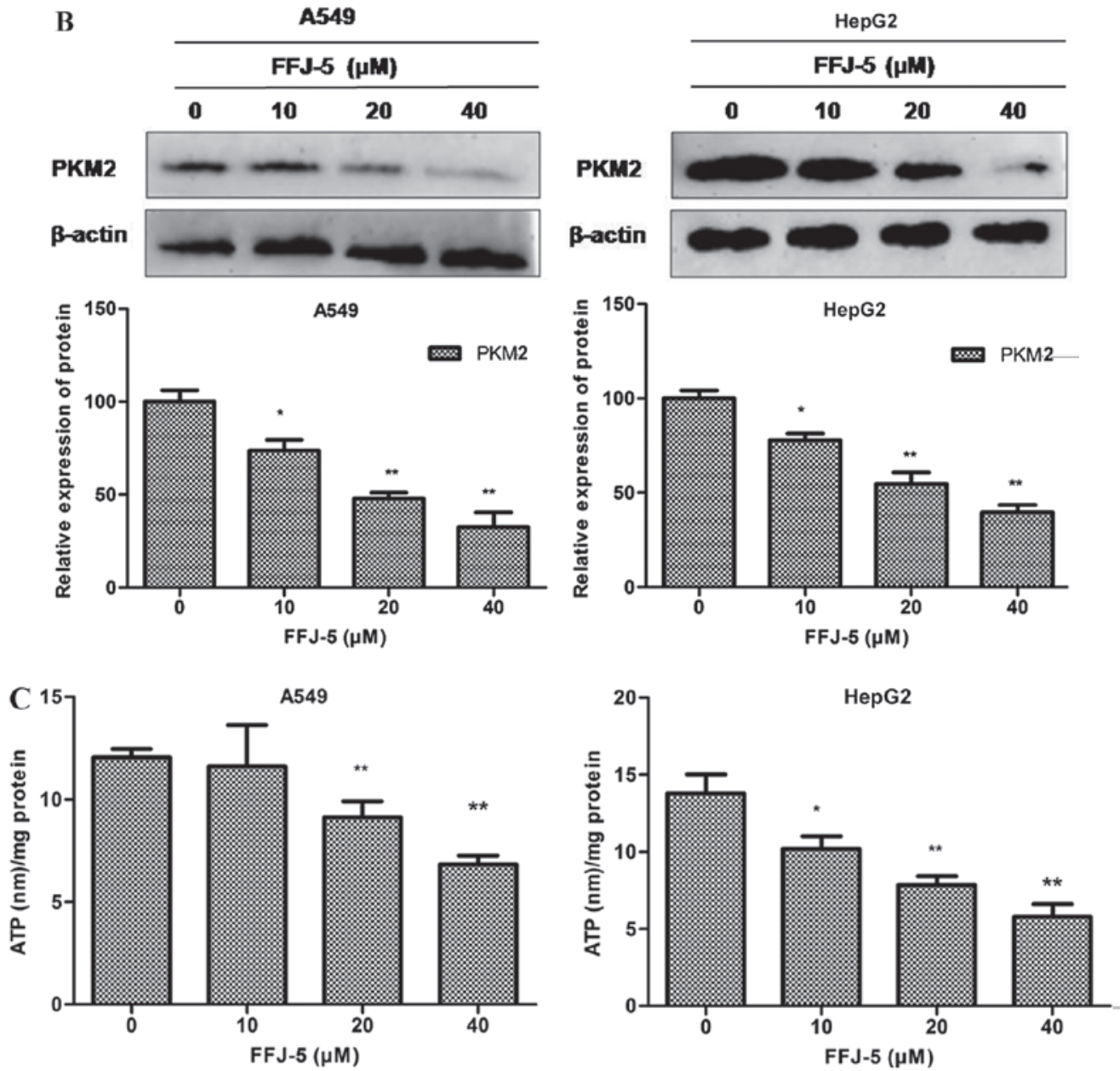

Figure 2. FFJ-5 inhibited the expression of PKM2 and reduced the ATP production in A549 and HepG2 cells. (A) Effect of FFJ-5 on the mRNA levels of PKM2. Reverse transcription-quantitative polymerase chain reaction demonstrated that FFJ-5 distinctly decreased the mRNA levels of PKM2. (B) Effect of FFJ-5 on the protein expression of PKM2. Western blotting revealed that FFJ-5 significantly inhibited the expression of PKM2. (C) ATP production analysis. FFJ-5 reduced the ATP production. The data represent the mean and standard deviation obtained from three independent experiments. " $\mathrm{P}<0.05$, ${ }^{* *} \mathrm{P}<0.01$. mRNA, messenger RNA; ATP, adenosine triphosphate; PMK2, pyruvate kinase isoenzyme M2.

microenvironment and regulating the cell cycle and cell apoptosis $(28,29)$. Naphthoquinone derivatives are specific inhibitors of PKM2 (14). FFJ-5, a novel naphthoquinone derivative, is a chemically modified compound based on the 

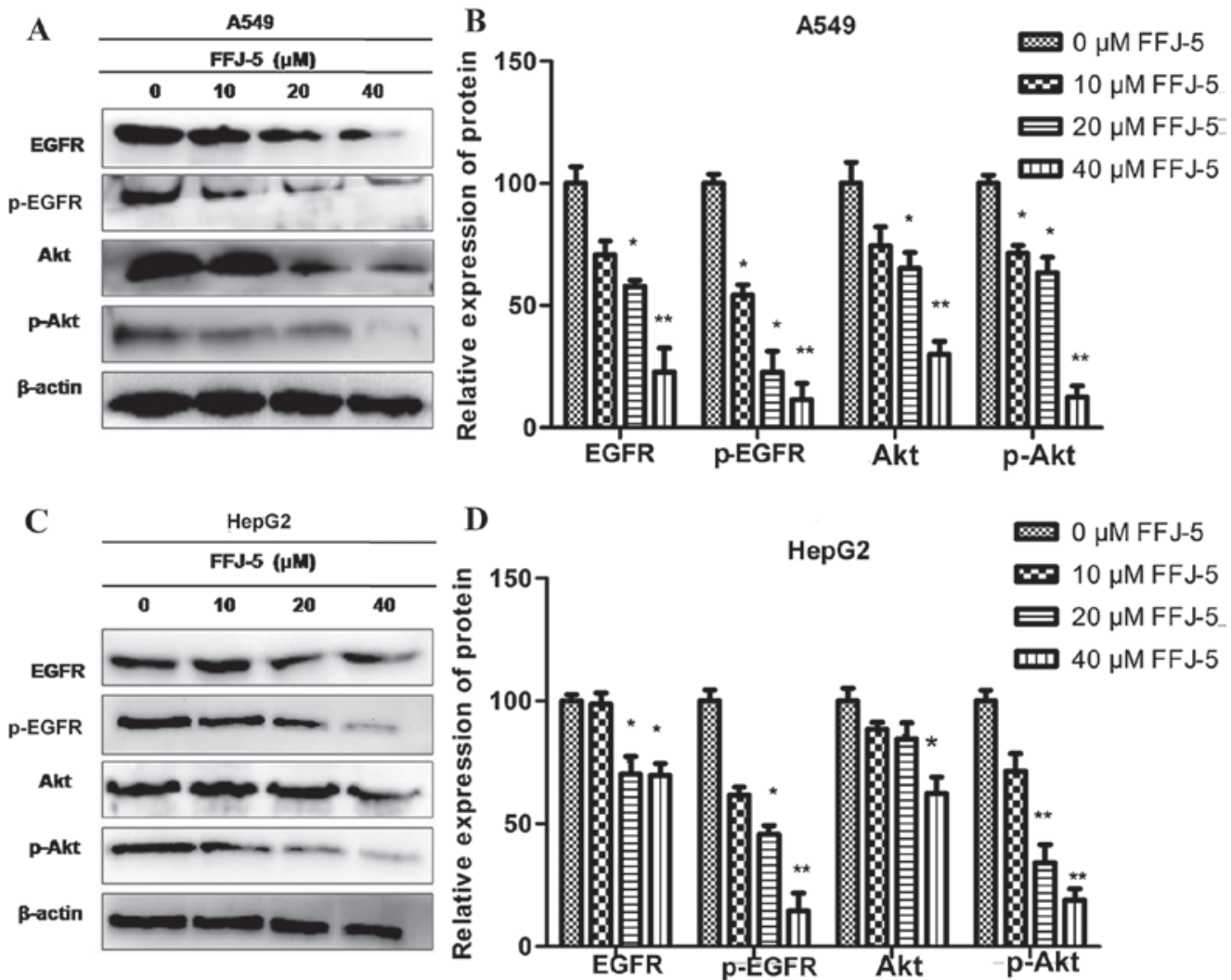

Figure 3. Effect of FFJ-5 on the expression and activity of EGFR and Akt. (A) Effect of treatment with different concentrations of FFJ-5 on the expression of EGFR, pEGFR, Akt and pAkt in A549 cells. (B) Statistical analysis of the expression levels of EGFR, pEGFR, Akt and pAkt in A549 cells following treatment with different concentrations of FFJ-5. (C) Effect of treatment with different concentrations of FFJ-5 on the expression of EGFR, pEGFR, Akt and pAkt in HepG2 cells. (D) Statistical analysis of the expression levels of EGFR, pEGFR, Akt and pAkt in HepG2 cells following treatment with different concentrations of FFJ-5. Western blotting revealed that FFJ-5 reduced the total protein levels of EGFR and Akt, and inhibited the phosphorylation of and EGFR and Akt. ${ }^{*} \mathrm{P}<0.05,{ }^{* *} \mathrm{P}<0.01$. EGFR, epidermal growth factor receptor; $\mathrm{p}$, phosphorylated; Akt, protein kinase $\mathrm{B}$.

A

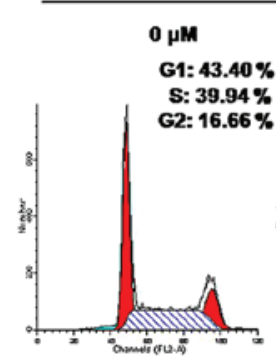

B
A549/FFJ-5

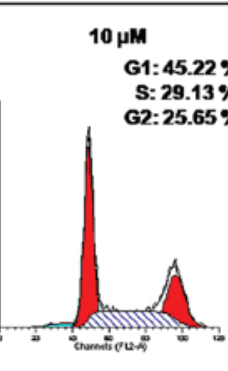

$20 \mu \mathrm{M}$

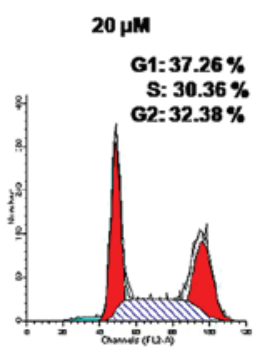

HepG2/FFJ-5
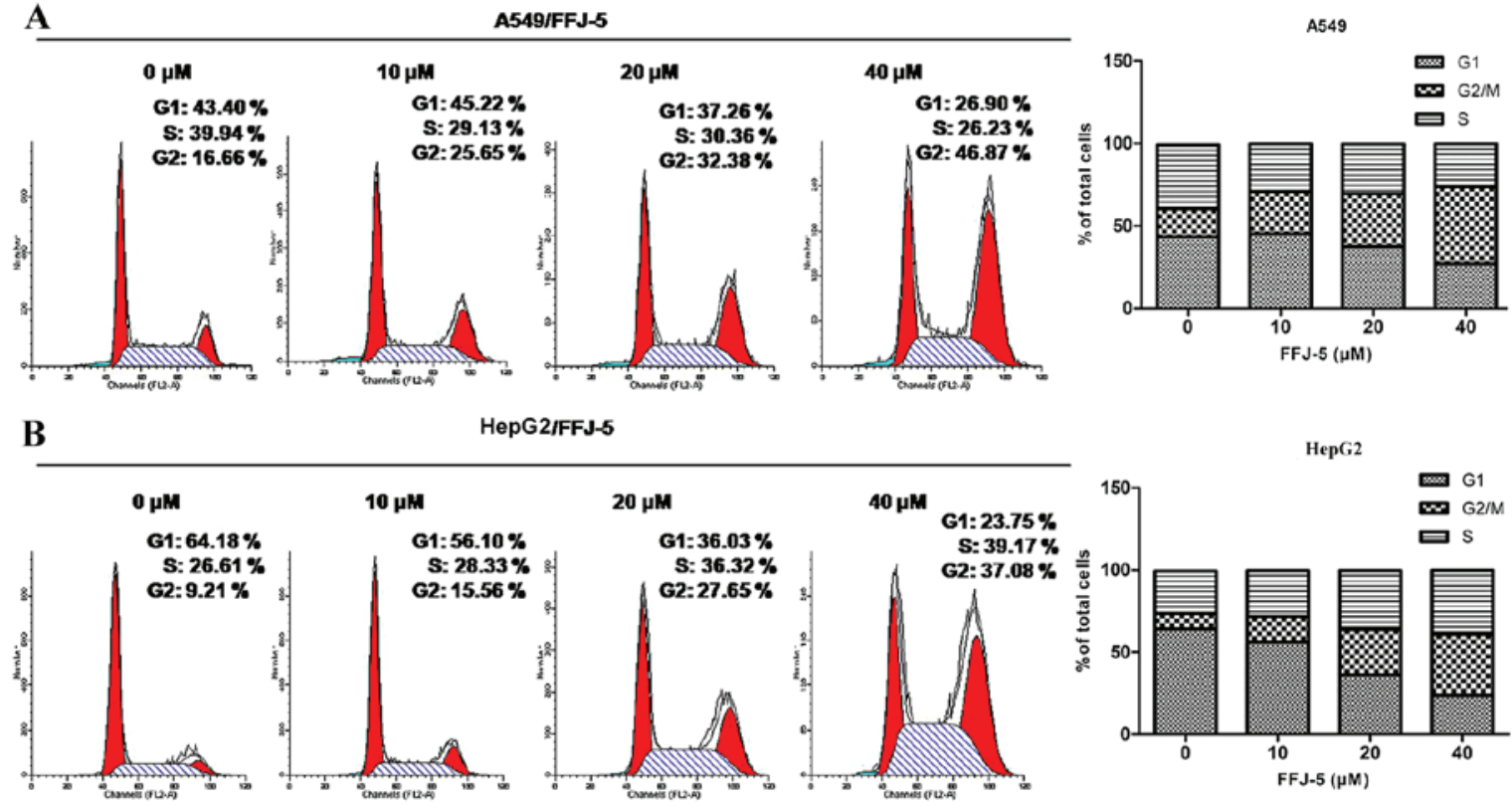

Figure 4. Cell cycle analysis. (A) Effect of treatment with different concentrations of FFJ-5 on the A549 cell cycle. (B) Effect of treatment with different concentrations of FFJ-5 on the HepG2 cell cycle. Cells were treated with FFJ-5 (0, 10, 20 and $40 \mu \mathrm{M})$ for $24 \mathrm{~h}$. Insets show the percentage distribution of cells in each cell cycle phase. FFJ-5 caused a significant accumulation of cells in the G2/M (4n DNA content) phase in HepG2 cells and A549 cells.

structure of mollugin (2). In the present study, high expression of PKM2 was detected in A549 and HepG2 cells by western blotting, indicating the important role of glycolysis in the development of lung and liver cancer. Our study revealed that 
A
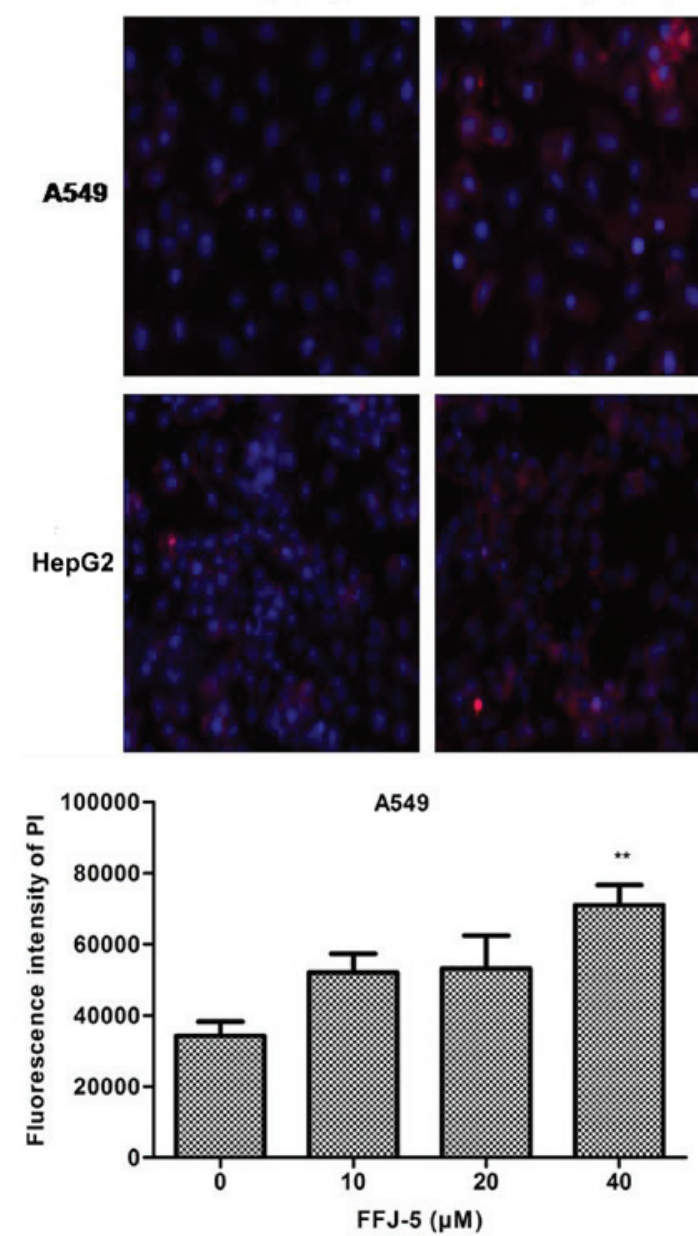

B

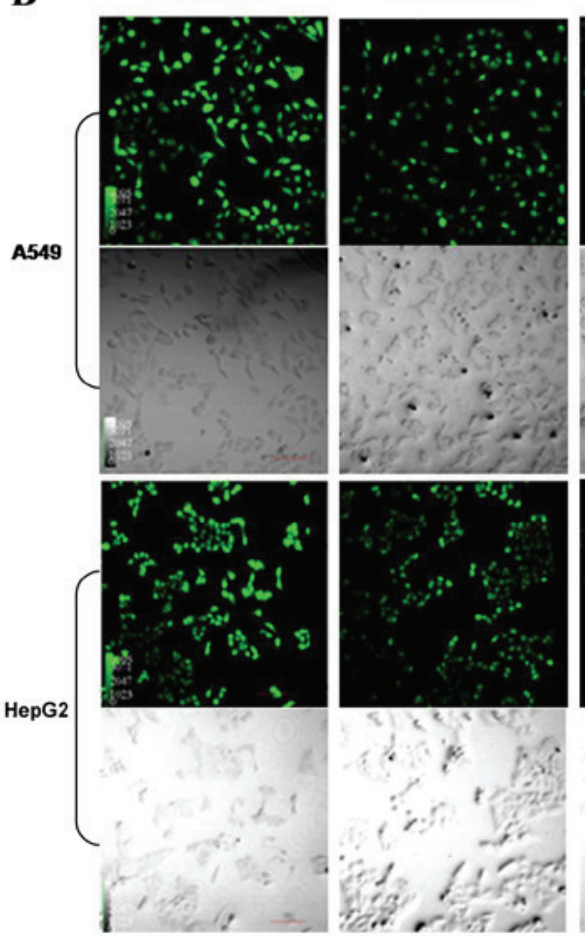

FFJ-5 (10 $\mu \mathrm{M})$
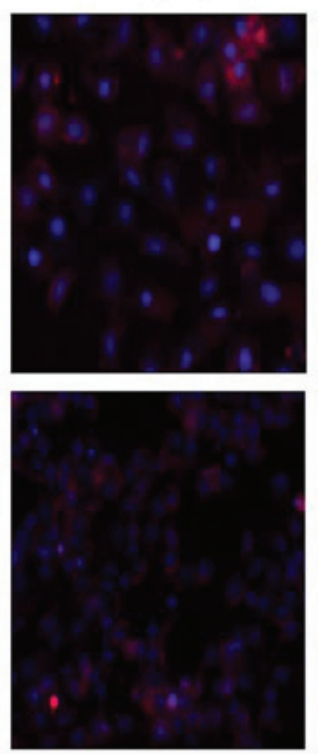

FFJ-5 (20 $\mu \mathrm{M})$
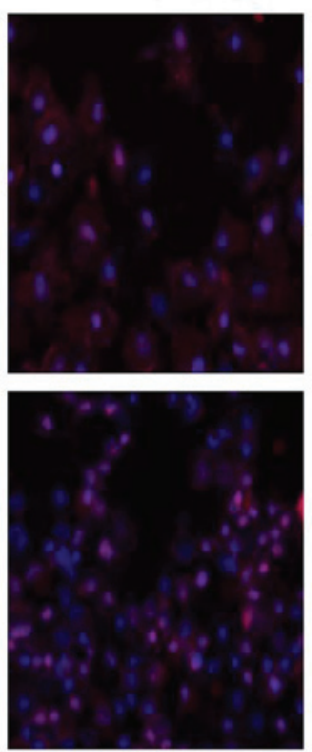

FFJ-5 (40 $\mu \mathrm{M})$
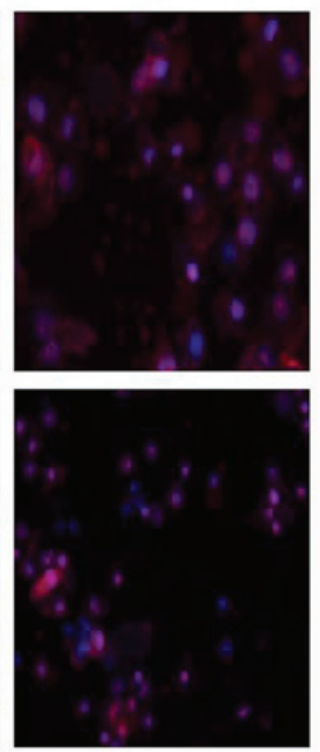

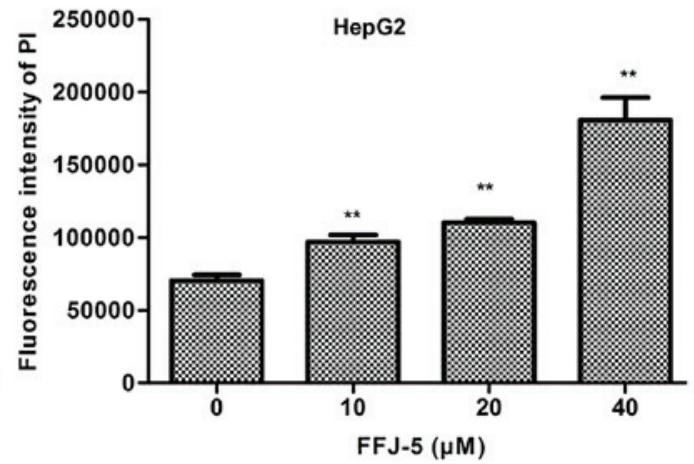

A549
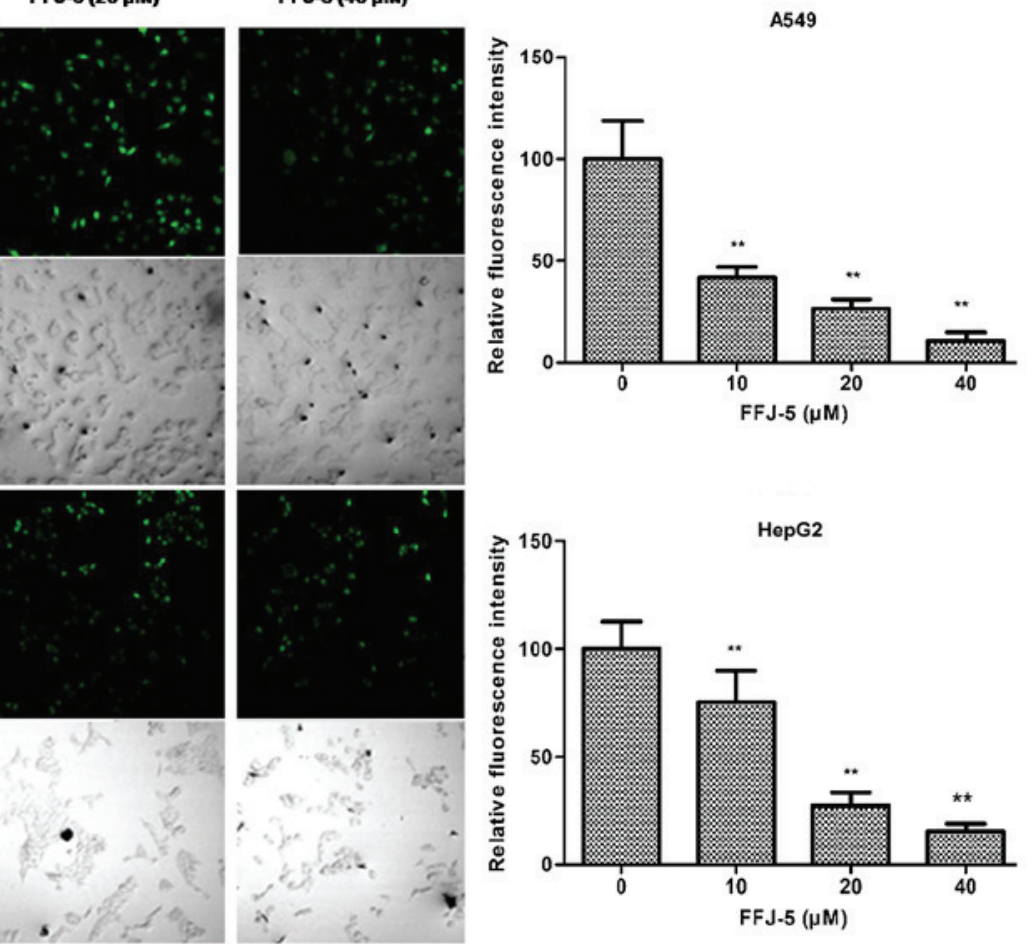

HepG2

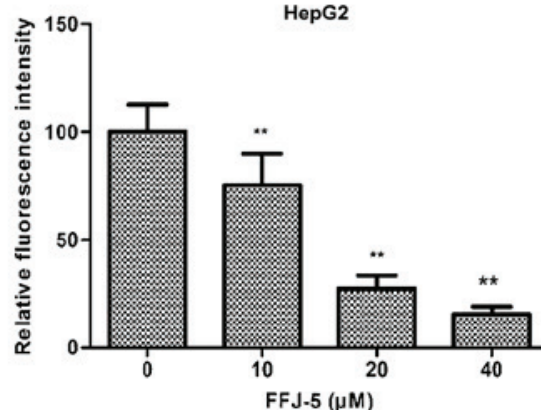

Figure 5. FFJ-5 reduced the apoptosis of A549 and HepG2 cells. (A) Effect of FFJ-5 on the fluorescence intensity of PI in A549 and HepG2 cells. Cells treated with $0,10,20$ and $40 \mu \mathrm{M}$ FFJ-5 for $48 \mathrm{~h}$ were incubated with $5 \mathrm{mg} / 1$ Hoechst 33342 for 10 min and with $5 \mathrm{mg} / 1 \mathrm{PI}$ for another $1 \mathrm{~h}$ in the dark. Then, the mean fluorescence intensity of PI was detected with an Array Scan VTI HCS 600-type high-content live cell imaging system. Magnification, x20. (B) Effect of FFJ-5 on the intracellular $\mathrm{pH}$ level. The intracellular $\mathrm{pH}$ level was verified by confocal scanning laser microscopy. A decreased intracellular $\mathrm{pH}$ level was observed in HepG2 and A549 cells treated with FFJ-5. The top images are fluorescent images; the bottom images are light images. Magnification, x20. 

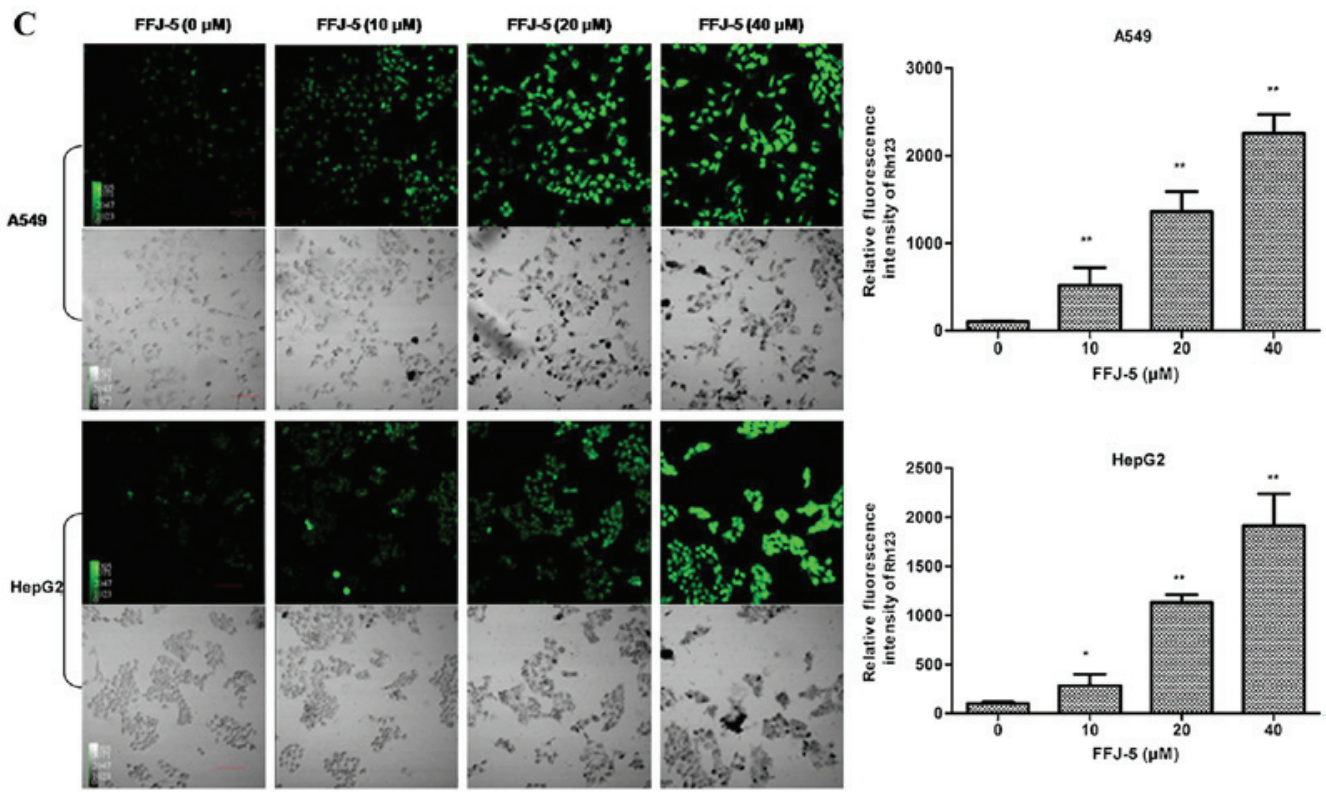

D
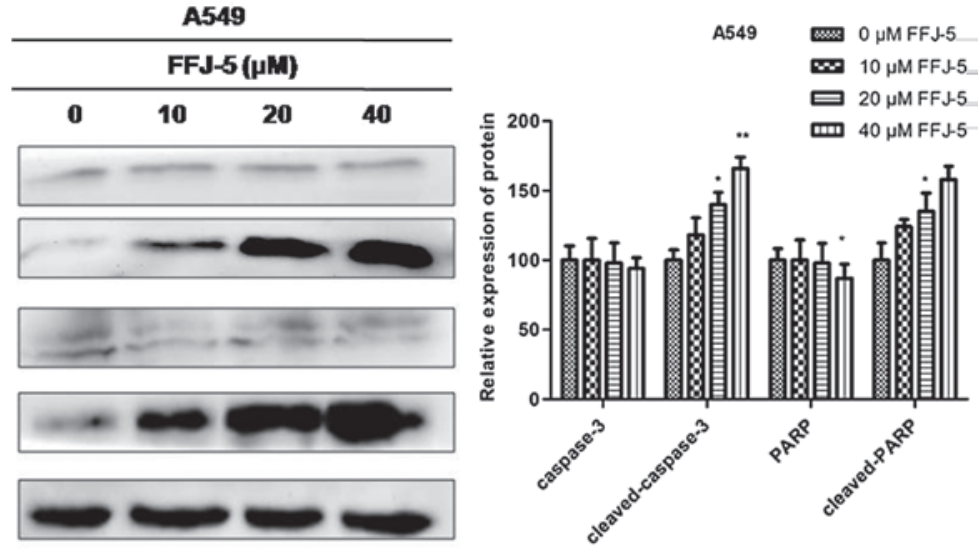

B-actin

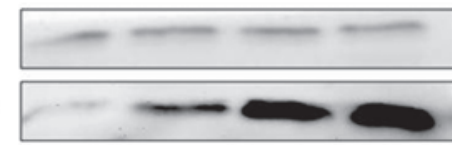

cleaved-caspase-3

PARP

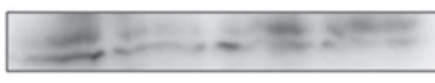

\section{cleaved-PARP}
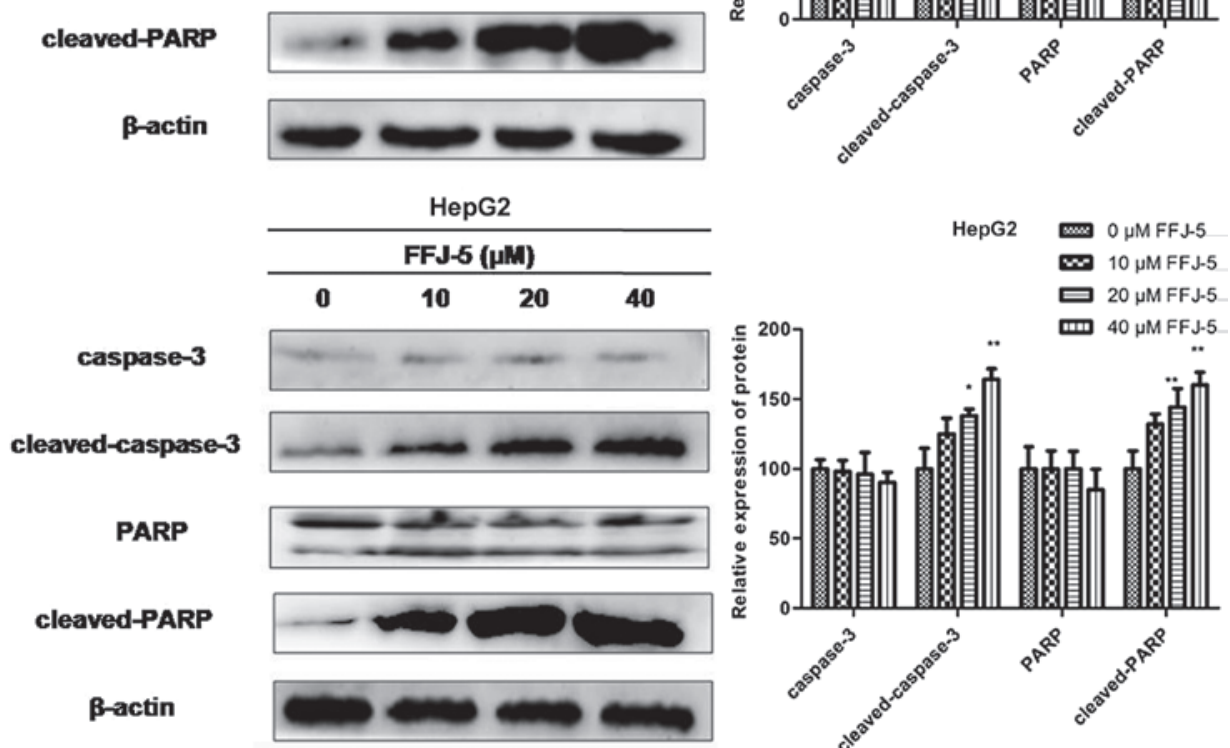

Figure 5. Continued. (C) FFJ-5 reduced the MMP in cancer cells. The change in MMP was detected with Rh123 staining. Increased Rh123 fluorescence intensity in cancer cells upon FFJ-5 incubation was observed. The top images are fluorescent images; the bottom images are light images. Magnification, $\mathrm{x} 20$. (D) FFJ-5 activated the caspase-3 cascade in cancer cells. Caspase-3, cleaved caspase-3, PARP and cleaved PARP levels were examined in A549 and HepG2 cells following FFJ-5 treatment for $48 \mathrm{~h}$ by western blotting. The data represent the mean and standard deviation obtained from three independent experiments. ${ }^{*} \mathrm{P}<0.05$ compared with the FFJ-5-untreated group. ${ }^{* *} \mathrm{P}<0.01$ compared with the FFJ-5-untreated group. PI, propidium iodide; MMP, mitochondrial membrane potential; Rh123, rhodamine 123; PARP, poly (ADP-ribose) polymerase.

FFJ-5 inhibited the expression of PKM2 protein, arrested the cell cycle in the G2/M phase and reduced the ATP production in A549 and HepG2 cells. Decreased intracellular pH levels and activated caspase cascade were also observed in cancer cells exposed to FFJ-5. FFJ-5 inhibited cancer cell growth and induced cancer cell apoptosis by blocking the EGFR-Akt-PKM2 signaling pathway, or via the synergistic action of EGFR, Akt and PKM2 proteins. These data suggest that FFJ-5 induced apoptosis in A549 and HepG2 cells by inhibiting PKM2 expression. 
In conclusion, the novel naphthoquinone compound FFJ-5 induces apoptosis in human lung adenocarcinoma A549 and human hepatoma HepG2 cells, and may be an effective anti-cancer candidate agent.

\section{Acknowledgements}

The authors would like to acknowledge the financial assistance provided by a grant from the Key Science and Technology Fund of Henan Province in China (grant no. 142300410128) to support the present project.

\section{References}

1. Shilpa PN, Venkatabalasubramanian S and Devaraj SN: Ameliorative effect of methanol extract of Rubia cordifolia in N-nitrosodiethylamine-induced hepatocellular carcinoma. Pharm Biol 50: 376-383, 2012.

2. Zhang L, Wang H, Zhu J, Xu J and Ding K: Mollugin induces tumor cell apoptosis and autophagy via the $\mathrm{PI} 3 \mathrm{~K} / \mathrm{AKT} / \mathrm{mTOR} / \mathrm{p} 70 \mathrm{~S} 6 \mathrm{~K}$ and ERK signaling pathways. Biochem Biophys Res Commun 450: 247-254, 2014.

3. Warburg O: On respiratory impairment in cancer cells Science 124: 269-270, 1956.

4. Cairns RA: Drivers of the Warburg phenotype. Cancer J 21: 56-61, 2015.

5. Warburg O: On the origin of cancer cells. Science 123: 309-314, 1956.

6. Koppenol WH, Bounds PL and Dang CV: Otto Warburg's contributions to current concepts of cancer metabolism. Nat Rev Cancer 11: 325-337, 2011.

7. Wu H,Li Z, Wang Y, Yang P, Li Z, Li H and Wu C: MiR-106b-mediated Mfn2 suppression is critical for PKM2 induced mitochondria fusion. Am J Cancer Res 6: 2221-2234, 2016.

8. Warner SL, Carpenter KJ and Bearss DJ: Activators of PKM2 in cancer metabolism. Future Med Chem 6: 1167-1178, 2014.

9. Iqbal MA, Gupta V, Gopinath P, Mazurek S and Bamezai RN: Pyruvate kinase M2 and cancer: An updated assessment. FEBS Lett 588: 2685-2692, 2014.

10. Chu B, Wang J, Wang Y and Yang G: Knockdown of PKM2 induces apoptosis and autophagy in human A549 alveolar adenocarcinoma cells. Mol Med Rep 12: 4358-4363, 2015.

11. Fendri A, Kontos CK, Khabir A, Mokdad-Gargouri R, Ardavanis A and Scorilas A: Quantitative analysis of BCL2 mRNA expression in nasopharyngeal carcinoma: An unfavorable and independent prognostic factor. Tumour Biol 31: 391-399, 2010.

12. Chen M, Zou X, Luo H, Cao J, Zhang X, Zhang B and Liu W: Effects and mechanisms of proton pump inhibitors as a novel chemosensitizer on human gastric adenocarcinoma (SGC7901) cells. Cell Biol Int 33: 1008-1019, 2009.

13. Ren J, Cheng H, Xin WQ, Chen X and Hu K: Induction of apoptosis by 7-piperazinethylchrysin in HCT-116 human colon cancer cells. Oncol Rep 28: 1719-1726, 2012.

14. Chen J, Jiang Z, Wang B, Wang Y and Hu X: Vitamin K(3) and $\mathrm{K}(5)$ are inhibitors of tumor pyruvate kinase M2. Cancer Lett 316: 204-210, 2012.
15. Yang W, Xia Y, Cao Y, Zheng Y, Bu W, Zhang L, You MJ, Koh MY, Cote G, Aldape K, et al: EGFR-induced and PKCe monoubiquitylation-dependent $\mathrm{NF}-\kappa \mathrm{B}$ activation upregulates PKM2 expression and promotes tumorigenesis. Mol Cell 48: 771-784, 2012.

16. Fan FT, Shen CS, Tao L, Tian C, Liu ZG, Zhu ZJ, Liu YP, Pei CS, Wu HY, Zhang L, et al: PKM2 regulates hepatocellular carcinoma cell epithelial-mesenchymal transition and migration upon EGFR activation. Asian Pac J Cancer Prev 15: 1961-1970, 2014.

17. Jiang Y, Li X, Yang W, Hawke DH, Zheng Y, Xia Y, Aldape K, Wei C, Guo F, Chen Y and Lu Z: PKM2 regulates chromosome segregation and mitosis progression of tumor cells. Mol Cell 53: 75-87, 2014.

18. Flint TR, Janowitz T, Connell CM, Roberts EW, Denton AE, Coll AP, Jodrell DI and Fearon DT: Tumor-induced IL-6 reprograms host metabolism to suppress anti-tumor immunity. Cell Metab 24: 672-684, 2016.

19. Warburg O, Wind F and Negelein E: The metabolism of tumors in the body. J Gen Physiol 8: 519-530, 1927.

20. Gao JL and Chen YG: Natural compounds regulate glycolysis in hypoxic tumor microenvironment. Biomed Res Int 2015: 354143, 2015.

21. Li C, Zhao Z, Zhou Z and Liu R: PKM2 promotes cell survival and invasion under metabolic stress by enhancing warburg effect in pancreatic ductal adenocarcinoma. Dig Dis Sci 61: 767-773, 2016.

22. Justus CR, Sanderlin EJ and Yang LV: Molecular connections between cancer cell metabolism and the tumor microenvironment. Int J Mol Sci 16: 11055-11086, 2015.

23. Fan SH, Wang YY, Wu ZY, Zhang ZF, Lu J, Li MQ, Shan Q, Wu DM, Sun CH, Hu B and Zheng YL: AGPAT9 suppresses cell growth, invasion and metastasis by counteracting acidic tumor microenvironmentthrough KLF4/LASS2/V-ATPase signaling pathway in breast cancer. Oncotarget 6: 18406-18417, 2015.

24. Zheng J: Energy metabolism of cancer: Glycolysis versus oxidative phosphorylation (Review). Oncol Lett 4: 1151-1157, 2012.

25. Tan X, Ye H, Yang K, Chen D and Tang H: Circadian rhythm variation of the clock genes Perl and cell cycle related genes in different stages of carcinogenesis of buccal mucosa in animal model. Zhonghua Kou Qiang Yi Xue Za Zhi 50: 392-398, 2015 (In Chinese).

26. Zheng L, Roeder RG and Luo Y: S phase activation of the histone $\mathrm{H} 2 \mathrm{~B}$ promoter by OCA-S, a coactivator complex that contains GAPDH as a key component. Cell 114: 255-266, 2003.

27. Lee KM, Nam K, Oh S, Lim J, Lee T and Shin I: ECM1 promotes the Warburg effect through EGF-mediated activation of PKM2. Cell Signal 27: 228-235, 2015.

28. Papadaki C, Sfakianaki M, Lagoudaki E, Giagkas G, Ioannidis G, Trypaki M, Tsakalaki E, Voutsina A, Koutsopoulos A, Mavroudis D, et al: PKM2 as a biomarker for chemosensitivity to front-line platinum-based chemotherapy in patients with metastatic non-small-cell lung cancer. Br J Cancer 111: 1757-1764, 2014

29. Chen Z, Wang Z, Guo W, Zhang Z, Zhao F, Zhao Y, Jia D, Ding J, Wang $\mathrm{H}$, Yao M, et al: TRIM35 interacts with pyruvate kinase isoform M2 to suppress the Warburg effect and tumorigenicity in hepatocellular carcinoma. Oncogene 34: 3946-3956, 2015. 